\title{
OPTICAL MONITORING OF THE GAMMA-RAY LOUD
}

BL LAC OBJECT S5 0716+714

G. LATINI, S. BOSIO, G. DE FRANCESCO, M. VILLATA

G. GHISELLINI AND C.M. RAITERI

Osservatorio Astronomico di Torino, Pino Torinese, Italy

G. TOSTI AND M. FIORUCCI

Università di Perugia, Via Pascoli, I-06100 Perugia, Italy

AND

M. MAESANO, E. MASSARO, F. MONTAGNI AND R. NESCI

Istituto Astronomico, Università La Sapienza, Roma, Italy

\section{Observations}

We monitored the $\gamma$-ray loud BL Lac object S5 $0716+714$ between November 1994 and April 1995 (J.D. 2449671-2449838), from the Observatories of Torino, Perugia and Roma. The source was always active in the $R$ and $B$ bands, showing a particularly high optical state during the period of the CGRO pointing (February 14-28, 1995). The lightcurve in the $R$ band is presented in Fig. 1. The complete set of observations is discussed in Ghisellini et al. (1995).

Fast variations are superimposed to longer trends. For the few well sampled peaks in the light curves, the rising and decaying time scales are equal, without 'plateau'. Moreover, the spectral index seems to respond to short time variations of the source, and is rather insensitive to long trends. There is an almost one-to-one correspondence between the changes in relative flux and those of the spectral index $\alpha_{B R}$ : the spectrum is flatter during local maxima of the flux.

2. Synchrotron Self-Compton model: expected $\gamma$-ray emission

A simple homogeneous, one-zone SSC model can fit the data, with $B=0.8$ $\mathrm{G}, \delta=13$ and $R=3 \times 10^{16} \mathrm{~cm}$. The relativistic electron distribution is a 

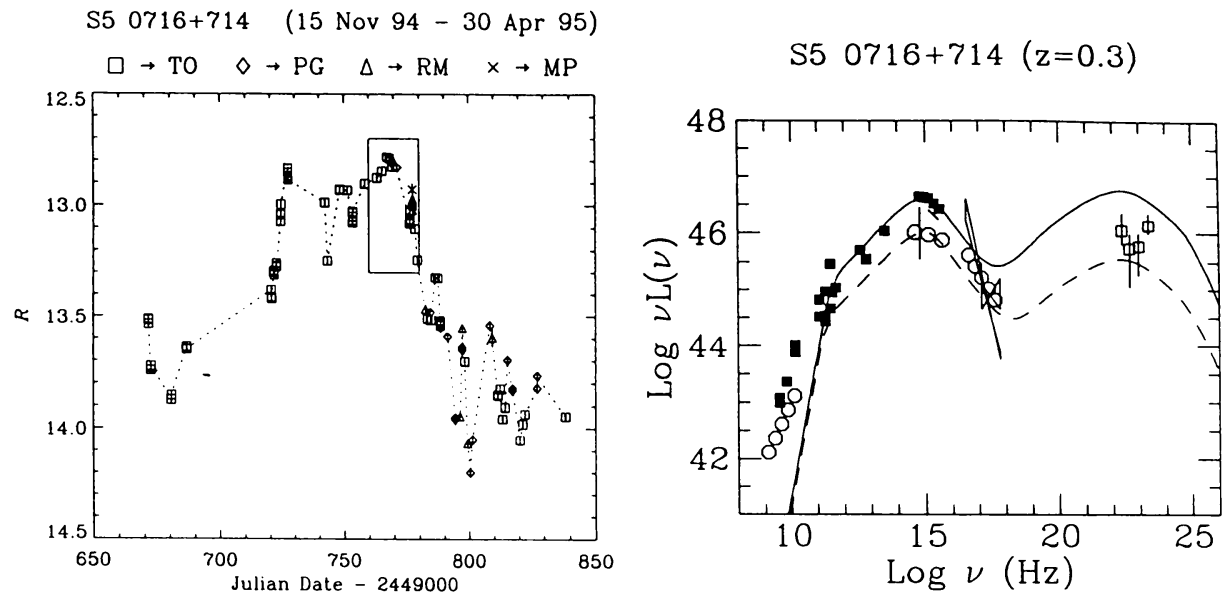

Figure 1. (left) Lightcurve in the $R$ band of 0716+714: the box shows the observations simultaneous with the GRO pointing. (right) Overall spectrum (assuming a redshift $z=0.3$, Schalinski et al. 1992) of S5 $0716+714$ from radio to $\gamma$-rays. The solid symbols in the optical ( $B, V$ and $R$ ) and UV correspond to data simultaneous (Feb. 27 and Mar. $1,1995)$ with the period of the GRO pointing, whose data are not public yet. Empty symbols refer to the spectrum published by Wagner et al. (1995). Solid and dashed lines refer to an homogeneous SSC model (see text).

broken power law with the break at $\gamma_{b}=5 \times 10^{3}$, producing a break of the synchrotron spectrum at $\sim 10^{15} \mathrm{~Hz}$. The resulting spectrum is shown in Fig. 1 as the solid line. The low optical state has been fitted by changing only the normalization of the electron distribution by a factor 4 (dashed line). This results in a decrease of the synchrotron luminosity of the same factor, while the Compton luminosity decreases quadratically. Note that:

In this one-zone model, the emission is predicted to vary simultaneously at all frequencies between the $\mathrm{mm}$ band up to the $\mathrm{GeV}$ band, although the amplitude of the synchrotron variations should be smaller than the Compton ones.

We predict that EGRET, in the observation of February 1995, should have observed the source in a very bright state, a factor between 3 and 10 above the flux level of the previous detection.

The plotted $\gamma$-ray data are not simultaneous with the low optical state.

\section{References}

Ghisellini G. et al., 1995, in preparation

Quirrenbach A. et al., 1991, ApJ, 372, L71

Schalinski C.J. et al., 1992, in Variability in Blazars, eds. E. Valtaoja \& M. Valtonen, (CUP), p. 221

von Montigny C. et al., 1995, ApJ, 440, 525

Wagner $S$. et al., 1995, $A J$, in press 